\title{
Adjetivos Subjetivos sob a abordagem da Gramática Discursivo-Funcional
}

\author{
George Henrique Nagamura \\ Universidade Federal de São Carlos (UFSCar), São Carlos, São Paulo, Brasil \\ genaga.trad@gmail.com
}

DOI: http://dx.doi.org/10.21165/el.v46i1.1575

\begin{abstract}
Resumo
O presente artigo tem como objetivo apresentar uma proposta para o tratamento de adjetivos subjetivos na Gramática Discursivo-Funcional (FDG). O artigo parte de uma discussão sobre o tratamento da modalidade subjetiva e os testes gramaticais empregados para essa distinção. $\mathrm{O}$ conceito de subjetividade proposto baseia-se nesses testes gramaticais, bem como na distinção feita por De Smet e Verstraete (2006) de diferentes tipos de subjetividade. Similarmente ao conceito desses autores, a proposta feita aqui distingue o que será chamado de Subjetividade Inerente, que permeia todos os usos da língua, e Subjetividade Gramatical, que se refere à codificação sistemática da Subjetividade Inerente. Além disso, será apresentada uma análise dos adjetivos subjetivos e, como resultado dessa análise, a noção de performatividade também é adicionada à definição de Subjetividade Gramatical.
\end{abstract}

Palavras-chave: subjetividade; modalidade; adjetivos; Gramática Discursivo-Funcional.

\section{Subjective Adjectives under the approach of Functional Discourse Grammar}

\begin{abstract}
The present work aims at presenting a proposal for the treatment of subjective adjectives in the Functional Discourse Grammar (FDG) framework. The article departs from a discussion on the treatment of Subjective Modals and the grammatical tests employed to make such distinction. The concept of subjectivity proposed is based on these grammatical tests as well as the distinction made by De Smet and Verstraete (2006) of different types of subjectivity. Similarly to their concept, the proposal here distinguishes what is to be called Inherent Subjectivity, which pervades all uses of language, and Grammatical Subjectivity, which is the systematic encoding of Inherent Subjectivity. Also, an analysis of subjective adjectives is presented, and, as a result, the notion of performativity is added to the definition of Grammatical Subjectivity.
\end{abstract}

Keywords: subjectivity; modality; adjectives; Functional Discourse Grammar.

\section{Introdução}

A Gramática Discursivo-Funcional (doravante GDF), de Hengeveld e Mackenzie (2008), sendo uma teoria relativamente nova, ainda não dispõe de um conceito definido de Subjetividade.

A subjetividade, entendida como a "expressão das atitudes do falante" por diversos pesquisadores (ex: TRAUGOTT, 2010), é de grande interesse para a GDF, uma vez que a "GDF tem como objetivo entender de que forma as unidades linguísticas se estruturam em termos do mundo que elas descrevem e das intenções comunicativas com as quais elas foram produzidas" (HENGEVELD; MACKENZIE, 2008, p. 2). A atitude do falante é parte das intenções comunicativas e, portanto, o entendimento de 
como essas atitudes influenciam a organização das unidades linguísticas cabe perfeitamente no escopo da GDF.

No presente artigo, parte-se da discussão do conceito de Modalidade Subjetiva e os testes gramaticais para sua identificação, como propostos por Hengeveld (1988; 2004) e as subsequentes críticas de Verstraete (2001) a respeito desses testes, na seção 1, seguida de uma apresentação do tratamento da subjetividade proposta por De Smet e Verstraete (2006), na seção 2, que conduzirão à proposta de definição da Subjetividade para essa teoria, na seção 3. O artigo encerra com a aplicação desse conceito para a distinção dos adjetivos subjetivos (seção 4) e, subsequentemente, as considerações finais.

\section{A Modalidade Subjetiva}

Narrog (2005), em seu artigo "On defining modality again”, analisa as principais concepções de modalidade, apontando, entre elas, a concepção baseada na noção de Subjetividade, influenciada, em grande medida, pela concepção oferecida por Lyons (1968, p. 308), como a marcação gramatical das "atitudes do falante". Nesse mesmo artigo, Narrog critica tal concepção, mostrando que, devido ao caráter vago da noção de subjetividade, os pesquisadores que adotam essa concepção arbitrariamente incluem ou excluem itens ou fenômenos linguísticos da categoria modal. Outro problema decorrente dessa concepção é a aparente incoerência de definir a modalidade como expressão da subjetividade (isto é, das atitudes do falante) e, ao mesmo tempo, distinguir modalidades subjetivas e objetivas.

Hengeveld (1988) prefere não oferecer um conceito para definir o fenômeno da modalidade como um todo. De acordo com o autor, "as diferentes distinções semânticas geralmente classificadas sob o rótulo 'modalidade' não parecem representar uma categoria semântica única e coerente" (HENGEVELD, 1988, p. 233). Em vez de oferecer uma definição de modalidade em geral, ele distingue três tipos de modalidade e discute as características de cada um deles. A modalidade inerente refere-se a "todos os meios linguísticos por meio dos quais um falante pode caracterizar a relação entre um participante em um Estado-de-Coisas e a realização potencial desse Estado-de-Coisas"1 (HENGEVELD, 1988, p. 233, tradução nossa). A modalidade objetiva refere-se a "todos os meios linguísticos por meio dos quais um falante pode avaliar a realidade de um Estado-de-Coisas em termos de seu conhecimento sobre os Estados-de-Coisas possíveis" ${ }^{2}$ (HENGEVELD, 1988, p. 233, tradução nossa). A modalidade epistemológica, por fim, refere-se a "todos os meios linguísticos por meio dos quais um falante pode expressar seu comprometimento com relação à verdade de uma proposição"'3 (HENGEVELD, 1988, p. 233, tradução nossa), também denominada nesse artigo como modalidade subjetiva. Essa distinção, à qual o autor se refere como alvo da avaliação, foi mantida no tratamento da modalidade na GDF (com os rótulos

\footnotetext{
${ }^{1}$ All those linguistic means through which $\mathrm{S}$ can characterize the relation between a participant in a SoA and the potential actualization of that SoA.

2 All those linguistic means through which $\mathrm{S}$ can evaluate the actuality of a SoA in terms of his knowledge of possible SoA's.

${ }^{3}$ All those linguistic means through which $\mathrm{S}$ can express his commitment with regard to the truth of a proposition.
} 
modalidade orientada para o participante, modalidade orientada para o evento e modalidade orientada para a proposição).

Para a identificação da modalidade subjetiva, Hengeveld (1988, 1989, 2004) propõe a adoção de testes gramaticais, segundo os quais modais subjetivos não podem ser utilizados em contextos de interrogação, não podem ser negados, não podem ser utilizados nas prótases de condicionais e somente podem ser usados no tempo presente. Hengeveld (1988, p. 236-237) oferece os seguintes exemplos:

(01) *Possibly John will come?

*'Possivelmente John virá?

(02) *If possibly John will come, I am going home.

*'Se possivelmente John virá, eu irei para casa.

(03) *Impossibly John will come.

*'Impossivelmente John virá.

(04) It may/might be true.

'Pode/Podia ser verdade'

Como pode ser observado nos exemplos (01) a (03), os enunciados não são aceitos quando o modal subjetivo ocorre nos contextos de interrogação, condicionalidade e negação. O exemplo (04) mostra que o modal may em sua forma passada might não se refere a uma possibilidade no passado, mas a uma estratégia de mitigação do comprometimento estabelecido pelo modal subjetivo.

Verstraete (2001) faz uma crítica aos testes propostos por Hengeveld, sem, no entanto, descartá-los. Segundo Verstraete (2001), é possível a ocorrência dos modais subjetivos em contextos de interrogação e condicionalidade, porém, nesses casos, há um efeito ecoico no enunciado, em que o falante parece repetir a fala de seu interlocutor (VERSTRAETE, 2001, p. 1519):

(05) Well then, the skeptical reader may ask, if only some stories have themes, if those themes may be hard to sum up, and if readers will probably disagree in their summations, why bother to state themes?

'Então, o leitor cético pode perguntar, se somente algumas histórias têm temas, e se esses temas talvez sejam difíceis de resumir, e se leitores provavelmente irão discordar quanto aos seus resumos, por que se preocupar em estabelecer temas?'

(06) Casually, Frido said to one of the colleagues in his room - 'Has anybody seen the Chief of Staff since he returned? He must know what's going on.' 'Must he?' Frido met a hard stare.

'Casualmente, Frido disse a um dos colegas na sala - "Alguém viu o chefe de departamento desde que ele voltou? Ele deve saber o que está acontecendo". "Deve?" Frido foi encarado vigorosamente.

No exemplo (05), pode-se perceber que "o modal may não expressa a opinião do falante, que utiliza a construção condicional, mas simplesmente ecoa a opinião expressa ou implicada no discurso anterior" (VERSTRAETE, 2001, p. 1520), assim como o modal must em (06).

Verstraete (2001) atribui a esses comportamentos do modal subjetivo a noção de performatividade modal. Da mesma forma que performativos tradicionais, como a forma eu prometo, trazem à existência uma ação (nesse caso, de promessa), os modais 
subjetivos trazem à existência uma posição de comprometimento do falante no momento de fala. Por esse motivo, o falante, ao utilizar um modal subjetivo, não pode trazer à existência um comprometimento e, ao mesmo tempo, questionar a existência desse comprometimento, negá-lo, ou colocá-lo na forma de hipótese. Por ser ligado ao momento da fala, essa avaliação não pode ocorrer no tempo passado ou futuro.

Nesta seção, foi apresentada a noção de testes gramaticais para a identificação dos modais subjetivos, fundamentada na noção de performatividade modal, de Verstraete (2001). A seguir, será apresentada outra forma de teste gramatical para a identificação da subjetividade, desta vez, com relação aos adjetivos.

\section{Adjetivos Subjetivos segundo De Smet e Verstraete (2006)}

Reconhecendo a dificuldade de se trabalhar com a noção de subjetividade, De Smet e Verstraete (2006) propõem a divisão da subjetividade em Subjetividade Pragmática e Subjetividade Semântica que, por sua vez, é dividida em Subjetividade Semântica Ideacional e Subjetividade Semântica Interpessoal. Segundo os autores, a Subjetividade Pragmática seria inerente à língua e presente em todo e qualquer uso. Um exemplo de subjetividade pragmática seria o uso de palavras como alto ou honesto. $\mathrm{O}$ uso dessas palavras sempre envolve a perspectiva do falante e os motivos que o levam a escolhê-las. Em outras palavras, a subjetividade está no uso dessas palavras.

Na Subjetividade Semântica, por outro lado, a subjetividade não é apenas algo resultante de seu uso, mas inerente ao significado da palavra, sendo o resultado de uma mudança de significado, de características mais concretas e externas para características mais abstratas e internas ao indivíduo. Os autores oferecem como exemplos de Subjetividade Semântica os adjetivos avaliativos leuk ("agradável") e dom ("estúpido", "maldito"), em holandês flamenco, que se referem à atitude interna do falante, contrastando com seus significados historicamente anteriores, que se referem a propriedades "externas" ("morno" e "incapaz de falar", respectivamente).

Os exemplos leuk e dom também ilustram a distinção entre a Subjetividade Semântica Ideacional e a Subjetividade Semântica Interpessoal, respectivamente. Os termos "Ideacional"e "Interpessoal" têm como origem os termos utilizados por Halliday (1994), em que "Ideacional" se refere ao nível de organização da linguagem que se presta à representação do mundo extralinguístico, enquanto "Interpessoal" se refere ao nível de organização da linguagem que se presta ao posicionamento do falante com respeito à representação de si e de sua interação com seu interlocutor. Essas duas categorias seriam igualmente subjetivas no sentido de que expressam uma avaliação interna do falante, mas se comportam de forma distinta: o uso de leuk, por exemplo, em leuke mensen ("pessoas agradáveis"), serve para criar uma subcategoria de pessoas que têm uma certa propriedade (no caso, de serem agradáveis); em contraste, dom, como em domme toeristen ("malditos turistas"), não serve para criar uma subcategoria de indivíduos, e, em vez disso, serve para realizar a atuação de uma posição (negativa, neste caso) do falante com relação a uma instância dessa categoria.

Além dessa diferença de funções, os autores propõem que Subjetividade Interpessoal apresenta restrições morfossintáticas, não podendo ser modificada em termos de gradação, nem por intensificadores e não pode ser utilizada predicativamente, como se observa nos exemplos a seguir (DE SMET; VERSTRAETE, 2006, p. 375): 
a. Met de domme ministers werd snel komaf gemaakt onder het nieuwe regime.

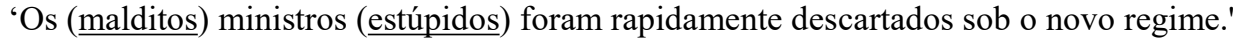

b. Met de domste ministers werd snel komaf gemaakt onder het nieuwe regime.

'Os ministros mais estúpidos foram rapidamente descartados sob o novo regime.'

c. Met de echt domme ministers werd snel komaf gemaakt onder het nieuwe regime.

'Os ministros realmente estúpidos foram rapidamente descartados sob o novo regime.'

d. De ministers met wie snel komaf werd gemaakt onder het nieuwe regime waren dom.

'Os ministros que foram rapidamente descartados sob o novo regime eram estúpidos'

O exemplo (07a) mostra que, em contextos sem as restrições mencionadas, dom pode tanto significar "maldito", como "estúpido" (isto é, sem inteligência). Nos contextos de gradação, intensificação e predicação, por outro lado, dom somente pode significar "estúpido". Essas restrições não se aplicam a leuk, cujo significado permanece o mesmo em todos os contextos:

a. Die leuke film heb ik gisteren al gezien.

'Aquele filme agradável eu já assisti ontem.'

b. Die leukste film heb ik gisteren al gezien.

'Aquele filme agradabilíssimo eu já assisti ontem.'

c. Die erg leuke film heb ik gisteren al gezien.

'Aquele filme realmente agradável eu já assisti ontem.'

d. Die film die ik gisteren heb gezien was leuk.

'O filme que assisti ontem foi agradável.'

Outra diferença gramatical, apontada por De Smet e Verstraete (2006), entre dom e leuk é que, em contextos negativos, o escopo da negação do SN que contém dom recai somente sobre o núcleo do sintagma (09a), ou então entende-se que dom se refere ao seu significado objetivo ('não inteligente') (09c), enquanto leuk pode manter seu significado subjetivo ('agradável'), mesmo quando o escopo da negação recai sobre a qualidade (09b):

(09) a. Wees gerust, mijn zus is geen domme advocaat. Ze is een beeldhouwster.

'Não se preocupe, minha irmã não é uma maldita advogada. Ela é uma escultora.'

b. Helaas, mijn zus is geen leuke advocaat. De getuigen zijn altijd als de dood voor haar.

'Infelizmente, minha irmã não é uma advogada agradável. As testemunhas sempre têm medo dela.'

c. Wees gerust, mijn zus is geen domme advocaat. Ze heeft nog nooit een zaak verloren.

'Não se preocupe, minha irmã não é uma advogada burra. Ela nunca perdeu um caso.'

De acordo com De Smet e Verstraete (2006), essas características gramaticais são reflexos da diferença de função entre os adjetivos subjetivos ideacionais e interpessoais: uma vez que o adjetivo interpessoal não se presta a criar uma subcategoria de pessoas que têm uma certa propriedade, seria também impossível especificar essa propriedade em termos de gradação e intensidade, assim como também seria impossível predicar essa propriedade ou ainda negá-la.

$\mathrm{Na}$ próxima seção, essas propostas de classificação da subjetividade serão levadas em conta para a proposta de conceito de subjetividade na GDF. 


\section{O Conceito de Subjetividade na GDF}

Uma das maiores dificuldades com relação ao estudo da subjetividade se deve ao fato de a noção de subjetividade ser, em geral, demasiadamente vaga ou de escopo muito abrangente. Narrog (2005, p. 170, tradução nossa), ao tratar das dificuldades na aplicação do conceito de subjetividade à definição de modalidade, comenta:

[...] é desafiador oferecer uma definição de 'subjetividade' ou de atitudes do falante na língua que não envolva uma quantidade considerável de imprecisão. Um segundo problema é onde estabelecer o limite entre subjetivo e objetivo (ou atitudinal vs. nãoatitudinal), isto é, como determinar a atual extensão da categoria. ${ }^{4}$

Relacionada a essa dificuldade de precisão dos limites da subjetividade está a noção de subjetividade como algo intrínseco à linguagem, como a propagada por Benveniste (1976, p. 287), para quem a linguagem "é tão profundamente marcada pela expressão da subjetividade que nós nos perguntamos se, construída de outro modo, poderia ainda funcionar e chamar-se linguagem”. Para o autor, o simples adereçamento a um outro na fala constitui o eu por meio da linguagem. Dessa maneira, todo e qualquer uso da língua é considerado subjetivo.

Essa abrangência dos limites da subjetividade parece ter encontrado uma solução na proposta de De Smet e Verstraete (2006), discutida anteriormente. Ao separar a Subjetividade Pragmática da Subjetividade Semântica, os autores permitem a denominação de formas subjetivas, sem contradizer o caráter subjetivo da linguagem, relacionado, entre outros fatores, ao caráter dialógico da linguagem.

Contudo, a proposta dos autores não pode ser tão prontamente adotada para os estudos na GDF. A razão disso é que a distinção entre Subjetividade Pragmática e Subjetividade Semântica de De Smet e Verstraete (2006) baseia-se no processo histórico de mudança de significado pelo qual passam os itens linguísticos. Para a GDF, enquanto essas mudanças não causam uma alteração na estrutura da língua, elas não devem ser consideradas. Nas palavras de seus autores, "a GDF se limita a reflexões gramaticais de significados sociais" (HENGEVELD; MACKENZIE, 2008, p. 29). Para compreender, entretanto, o que significa essa limitação, será necessário explicar, resumidamente o funcionamento da GDF.

A estrutura da GDF é dividida em Níveis e Camadas. Cada Nível é estruturado de maneira diferente, com suas próprias Camadas, sempre organizadas hierarquicamente. São quatro os níveis descritos na GDF, a saber, o Interpessoal, o Representacional, o Morfossintático e o Fonológico. Tendo em vista os objetivos deste trabalho, a descrição será limitada aos Níveis Interpessoal e Representacional, níveis mais diretamente relacionados à manifestação da subjetividade. Basta saber, por hora, que os Níveis Morfossintático e Fonológico se encarregam da codificação do conteúdo gerado nos Níveis Interpessoal e Representacional e que esses dois níveis de codificação também possuem sua própria estrutura em camadas.

\footnotetext{
${ }^{4}[\ldots]$ it is challenging to give a definition of subjectivity or speakers attitudes in language that does not involve a fair amount of vagueness. The second, related issue is where to draw the borderline between subjective and objective (or attitudinal vs. non-attitudinal), that is, how to determine the actual extension of the category.
} 
O Nível Interpessoal captura todas as distinções que se referem à interação entre o falante e o ouvinte. A camada mais alta deste nível é o Movimento (M), que pode ser definido como uma contribuição autônoma para a interação em curso. Pode tanto pedir uma reação, quanto ser a própria reação a outro pedido. Um Movimento pode ser composto de um ou mais Atos Discursivos (A), que constituem a camada seguinte. Cada Ato Discursivo pode conter os seguintes componentes (todos em relação de equipolência): uma Ilocução $(\mathrm{F})$, o Falante $\left(\mathrm{P}_{\mathrm{S}}\right)$, o Ouvinte $\left(\mathrm{P}_{\mathrm{A}}\right)$ e o Conteúdo Comunicado $(\mathrm{C})$. Considerando a referência e a atribuição como ações pragmáticas, isto é, que provocam mudanças na informação pragmática do ouvinte, a GDF distingue também os Subatos de Referenciação (R) e Atribuição (T), como uma camada abaixo do Conteúdo Comunicado.

O Nível Representacional lida com o aspecto semântico das unidades linguísticas. Enquanto no Nível Interpessoal é realizada a evocação, no Nível Representacional é realizada a designação. O Nível Representacional inicia com a camada do Conteúdo Proposicional (p) e se refere a crenças, esperanças, e outros tipos de construções mentais. Os Conteúdos Proposicionais contêm Episódios (ep), conjuntos de Estados-de-Coisas (e) tematicamente coerentes, que mostram continuidade de tempo (t), local (1) e indivíduos (x). Os Estados-de-Coisas incluem eventos e estados que são caracterizados pelo fato de poderem ser localizados no tempo e poderem ser avaliados em termos de seu estatuto de realidade. Pode-se dizer, então, de um Estado-de-Coisas que ele (não) ocorreu em um determinado ponto ou intervalo de tempo. Um Estado-deCoisas é caracterizado por uma Propriedade Configuracional (f), que contém uma combinação de unidades semânticas que não estão em relação hierárquica entre si. As Propriedades Configuracionais constituem o inventário de esquemas de predicação relevantes para uma língua, permitidas de acordo com a valência qualitativa ou quantitativa, e são constituídas por categorias semânticas em relação não-hierárquica. Essas categorias podem ser Indivíduos (x), Propriedades Lexicais (f), Local (1), Tempo (t), Modo (m), Razão (r), ou Quantidade (q).

Retornando aos exemplos oferecidos por De Smet e Verstraete (2006), no tratamento sob a ótica da GDF, ambos os adjetivos alto e agradável (exemplos de subjetividade pragmática e subjetividade semântica ideacional, respectivamente) são representados na estrutura hierárquica como Propriedades Lexicais (f), modificadoras de Indivíduos (x). Em outras palavras, esses adjetivos não apresentam características gramaticais consideradas relevantes para a GDF.

Em contraste, a distinção realizada entre Subjetividade Semântica Ideacional e Subjetividade Semântica Interpessoal apresenta dados relevantes para o seu tratamento na GDF. Além das restrições gramaticais apresentadas por De Smet e Verstraete (2006), há uma diferença crucial entre adjetivos como agradável e maldito, a saber, a possibilidade de aplicação a um nome próprio.

Como se pode observar nos exemplos a seguir, somente o adjetivo maldito pode ser aplicado a nomes próprios:

(10) Maldito Bush!

(11) *Agradável Bush! 
Em sua representação na GDF, nomes próprios são considerados vazios de conteúdo semântico, o que significa que a modificação realizada pelo adjetivo maldito não se aplica ao Nível Representacional, mas sim ao Nível Interpessoal.

Assim, na GDF, enquanto adjetivos como alto ou agradável são considerados modificadores de Indivíduo (x), uma unidade do Nível Representacional, adjetivos como maldito são considerados modificadores de Subato Referencial (R), uma unidade do Nível Interpessoal.

A proposta de subdivisão da subjetividade, de De Smet e Verstraete (2006), contudo, parece ser adequada à GDF, com algumas alterações. Em primeiro lugar, dada a limitação da GDF a reflexões gramaticais de significados sociais, a subdivisão que deve ser feita da subjetividade é em termos das características gramaticais desses itens. Em segundo lugar, os rótulos aplicados por De Smet e Verstraete devem ser adaptados, para refletir melhor essa distinção e evitar confusões com termos já empregados na teoria. Por exemplo, o termo pragmático, embora descreva bem a noção de que esse tipo de subjetividade depende do uso, confunde-se com a definição de Interpessoal, da GDF, que se refere à interação entre o falante e seu interlocutor. Além disso, o termo Interpessoal de De Smet e Verstraete pode confundir-se com o termo Interpessoal da GDF, que se refere a um Nível da organização hierárquica. Uma vez que não há nada na definição baseada em diferenças gramaticais que impeça a manifestação da subjetividade no Nível Representacional, o termo Interpessoal parece inadequado para designar esse tipo de subjetividade.

Por esses motivos, proponho a divisão da subjetividade na GDF em Subjetividade Inerente e Subjetividade Gramatical. Identificados dessa maneira, a Subjetividade Inerente se refere a todo e qualquer uso da linguagem, incluindo aí o processo dialógico que estabelece um $e u$ em oposição a um outro, bem como o uso de adjetivos como alto, agradável, e até mesmo maldito, que, em medidas diferentes envolvem decisões do falante e expressam, também em diferentes medidas, sua realidade interna. A Subjetividade Gramatical, por outro lado, refere-se à sistematização gramatical de algumas dessas avaliações, como no caso de maldito, que apresenta restrições gramaticais que não se aplicam a adjetivos como alto ou agradável.

É preciso deixar claro que a Subjetividade Gramatical se inclui no domínio da Subjetividade Inerente, ou seja, uma vez que a Subjetividade Inerente se aplica a todo e qualquer uso da língua, obviamente, ela também se aplica à Subjetividade Gramatical. $\mathrm{O}$ adjetivo maldito, portanto, é considerado ao mesmo tempo expressão da Subjetividade Inerente e da Subjetividade Gramatical. Os adjetivos alto e agradável são expressão apenas da Subjetividade Inerente.

$\mathrm{Na}$ seção seguinte, serão examinados os testes gramaticais propostos por De Smet e Verstraete para os adjetivos e sua adequação para o estudo da subjetividade na GDF e a importância do conceito de performatividade. 


\title{
4. Os testes gramaticais para os Adjetivos Subjetivos e a noção de performatividade
}

Segundo Hengeveld (2008, p. 48), os modificadores de sintagmas nominais podem assumir as seguintes funções:

\author{
$\sum^{\mathrm{R}} \quad$ Atitude Subjetiva - Modificador de Subato de Referência (R) \\ $\sigma^{\mathrm{x}} \quad$ Modificação de referente - Modificador de Indivíduo (x) \\ $\sigma^{\mathrm{f}} \quad$ Modificação de referência - Modificador de Propriedade Lexical (f) \\ O autor oferece os seguintes exemplos de cada função (HENGEVELD, 2008, p.
}

49):

(12) Oh my god, the poor $\left(\sum^{\mathrm{R}}\right)$ doctor was going to just tell me the results!

'Meu Deus, o pobre doutor ia somente me dizer os resultados!'

(13) Had I run into the rarest of species, one most people would have thought was extinct in the western world: a poor $\left(\sigma^{\mathrm{x}}\right)$ doctor.

'Encontrara uma das espécies mais raras, uma que muitos pensavam estar extinta no mundo ocidental: um médico pobre.'

(14) A poor $\left(\sigma^{f}\right)$ doctor, dentist or nurse can cause huge harm to a patient in 16 minutes let alone 16 weeks.

'Um médico, dentista ou enfermeiro ruins podem causar grandes danos a um paciente em 16 minutos, que dirá em 16 semanas.'

Conforme dito anteriormente, De Smet e Verstraete (2006) propõem que os adjetivos subjetivos Interpessoais se distinguem dos adjetivos subjetivos Ideacionais por não poderem ser modificados em termos de gradação, intensificação, negação e por não poderem ser predicados.

Essas características, a princípio, parecem ser válidas como foi visto na seção 2, em que se mostra a diferença entre os adjetivos dom e leuk, do holandês flamenco.

Contudo, tomando como exemplo o adjetivo former do inglês ('antigo', 'que não é mais'), um modificador de Propriedade Lexical, pode-se notar que as mesmas restrições também se aplicam a esse tipo de modificador:

(15) a former neighbour

'um ex-vizinho, um antigo vizinho'

a. Mike is my former neighbour

'Mike é meu antigo vizinho'

b. *Mike is a more former neighbor than John.

'Mike é um vizinho mais antigo do que John'

c. *Mike is my very former neighbour

'Mike é meu muito antigo vizinho'

d. *The neighbour is former.

'O vizinho é antigo'

Os exemplos mostram como o adjetivo former também é restrito dos mesmos contextos que os modificadores de Subato Referencial. Uma vez que a modificação da 
Propriedade Lexical não exprime uma atitude do falante, e, assim, dificilmente seria considerada uma forma de expressão de subjetividade, parece seguro afirmar que os testes propostos por De Smet e Verstraete para os adjetivos não são adequados para realizar a identificação dos adjetivos subjetivos.

Como afirma Verstraete (2001), critérios gramaticais são problemáticos quando se aplicam sem entendimento do motivo para aplicá-los. De Smet e Verstraete (2006) explicam que os testes gramaticais aplicados mostram que o adjetivo dom ('maldito') não se presta mais à definição de um subtipo de entidade. O problema é justamente a equação realizada pelos autores entre não designar um subtipo de entidade e expressar a atitude do falante. Assim como dom, o modificador de Propriedade Lexical não designa um tipo de entidade, mas especifica que a propriedade ser vizinho não é mais válida.

Por outro lado, os testes de interrogação e negação se aplicam aos modificadores de Subato Referencial, distinguindo-os dos modificadores de Indivíduo e Propriedade Lexical. Como visto anteriormente, modificadores de Subato Referencial, como dom não podem ser negados. O mesmo não ocorre com modificadores de Propriedade Lexical:

(16) He is not a former martial artist. He still IS a martial artist.

'Ele não é um ex-lutador. Ele ainda É um lutador'

Nesse exemplo, pode-se notar que a propriedade former, ao indicar que a designação não mais se aplica, está sendo negada, como fica explícito na segunda parte do enunciado. Nos exemplos abaixo, é possível observar, nas respostas aos questionamentos, qual parte do enunciado está sendo interrogada, ou seja, pelo contraste entre antigo e atual em (17) e alta e baixo em (18), vê-se que a propriedade está em questionamento; em (19), o contraste na resposta somente pode ser entre advogado e professor, indicando que a designação está em questionamento. $\mathrm{O}$ estranhamento de (19B2) indica a inadequação do contraste entre maldito e agradável.

A: Is he a former neighbour?

'Ele é um antigo vizinho?'

B: No, he is a current one.

'Não, ele é um vizinho atual'

(18) A: Is he a tall person?

'Ele é uma pessoa alta?'

B: No, he is a short one.

'Não, ele é baixo'

(19) A: Is he a damn lawyer?

'Ele é um maldito advogado?'

B1: No, he is a teacher.

'Não, ele é um professor'

B2: ? No, he is very pleasant.

? 'Não, ele é muito agradável.' 
Os testes de condicionalidade e tempo presente, por sua vez, parecem não se aplicar aos adjetivos, já que, diferentemente da interrogação e negação, que podem focar apenas parte da informação no enunciado, a falta de comprometimento da condicional se aplica à proposição como um todo, e o tempo não pode ser aplicado a propriedades sem o auxílio da cópula.

Dessa forma, os testes gramaticais para os adjetivos subjetivos também revelam a relação entre a avaliação subjetiva e a performatividade do comprometimento, o que leva a uma definição mais precisa do conceito de subjetividade na GDF, que será realizada na seção seguinte.

\section{Considerações finais}

A análise apresentada na seção anterior mostrou a inadequação dos testes gramaticais propostos por De Smet e Verstraete (2006) para os adjetivos subjetivos e o sucesso da aplicação dos testes desenvolvidos para os modais subjetivos, diferenciando modificadores de Subato Referencial (considerados subjetivos), de modificadores de Indivíduo e Propriedade Lexical, revelando, assim, a conexão entre a subjetividade e a performatividade do comprometimento.

Lembrando, como o faz Verstraete (2001), que a aplicação dos testes gramaticais é problemática quando não se entende o motivo de sua aplicação, é necessário incluir, na definição de Subjetividade Gramatical, a noção de performatividade, o que leva às seguintes concepções: a Subjetividade na GDF é dividida em dois tipos, Subjetividade Inerente, que se refere ao caráter dialógico da linguagem, chamada assim por se entender que, em todo ato de enunciação, o falante se coloca como um eu que se dirige a um outro, expressando, mesmo em seus usos mais mundanos, seu ponto de vista, e Subjetividade Gramatical, que se refere à sistematização e formalização das expressões de atitude, ou seja, a avaliações necessariamente ligadas ao falante e ao momento de fala.

Acredito que o conceito de Subjetividade entendido dessa maneira apresenta diversas vantagens. Em primeiro lugar, restringe, pela adoção de testes gramaticais e da noção de performatividade, o objeto de pesquisa, permitindo uma distinção clara do que é considerado subjetivo e objetivo, em termos da Subjetividade Gramatical. Em segundo lugar, por meio da concepção de Subjetividade Inerente, não se descarta o caráter dialógico da linguagem e a noção de que todo e qualquer uso da língua envolve o falante. Por fim, por meio dessa distinção, é possível desfazer a incoerência existente entre a definição da modalidade como "forma de expressão das atitudes do sujeito", ou seja, de subjetividade, e, ao mesmo tempo, distinguir modalidades subjetivas e objetivas.

\section{REFERÊNCIAS}

BENVENISTE, E. Da subjetividade na linguagem. In: __. Problemas de linguística geral. Tradução de Maria da Glória Novak e Luiza Neri. São Paulo: Companhia Editora Nacional/Editora da Universidade de São Paulo, 1976. p. 284-293.

DE SMET, H.; VERSTRAETE, J. C. Coming to terms with subjectivity. Cognitive Linguistics, 17.3, p. 365-392, 2006. 
HALLIDAY, M. A. K. An Introduction to Functional Grammar. 2. ed. Londres: Arnold, 1994.

HENGEVELD, K. Illocution, mood and modality in a functional grammar of Spanish. Journal of Semantics, 6, p. 227-269, 1988.

Layers and operators in Functional Grammar. Journal of Linguistics, 25.1, p. 127-157, 1989.

Mood and Modality. In: BOOIJ, G.; LEHMANN, C.; MUGDAN, J. (Ed.). Morphology: A handbook on inflection and word formation. Berlim: Mouton de Gruyter, 2004. p. 1190-1202.

Prototypical and non-prototypical noun phrases in Functional Discourse Grammar. In: RIJKHOFF, J.; VELASCO, D. G. (Ed.). The Noun Phrase in Functional Discourse Grammar. Berlim: Mouton de Gruyter, 2008. p. 43-62.

HENGEVELD, K.; MACKENZIE, J. L. Functional Discourse Grammar: a typologically based theory of language structure. Oxford: Oxford University Press, 2008.

LYONS, J. Introduction to theoretical linguistics. Londres: Cambridge University Press, 1968.

NARROG, H. On defining modality again. Language Sciences, 27.2, p. 165-192, 2005.

TRAUGOTT, E. C. (Inter)subjectivity and (inter)subjectification: a reassessment. In: CUYCKENS, H.; DAVIDSE, K.; VANDELOTTE, L. (Ed.). Subjectification, Intersubjectification and Grammaticalization. Berlim: Mouton de Gruyter, 2010. p. 2971.

VERSTRAETE, J. C. Subjective and objective modality: Interpersonal and Ideational functions in the English modal auxiliary system. Journal of Pragmatics, 33, p. 1505$1528,2001$.

Recebido em: 15/08/2016

Aprovado em: 28/10/2016 